\title{
Impact of the Primary Resource Occupancy Information on the Performance of Cognitive Radio Networks with VoIP Traffic
}

\author{
S. Lirio Castellanos-Lopez ${ }^{\mathrm{a}}$, \\ Felipe A. Cruz-Pérez ${ }^{a}$ \\ ${ }^{a}$ Elect. Eng. Dept., CINVESTAV-IPN \\ Mexico City, Mexico \\ \{lirio, facruz\}@cinvestav.mx
}

\author{
Mario E. Rivero-Angeles ${ }^{\mathrm{b}}$ \\ b Telematics Department \\ UPIITA-IPN \\ Mexico City, Mexico \\ mriveroa@ipn.mx
}

\author{
Genaro Hernandez-Valdez ${ }^{\mathrm{c}}$ \\ ${ }^{\mathrm{c}}$ Electronics Department \\ UAM-A \\ Mexico City, Mexico \\ ghv@correo.azc.uam.mx
}

\begin{abstract}
In this paper, the relevance of considering the primary network resource occupancy information on the admission criterion of new secondary VoIP sessions in cognitive radio networks (CRNs) is investigated. In particular, the performance of two different call admission control (CAC) strategies whose admission criterion is based either on the total number of primary and secondary sessions or only on the number of sessions of secondary users is compared. System performance is evaluated in terms of the most relevant quality of service metrics for VoIP traffic at both call and packet level. Numerical results clearly show that including primary resource occupancy information on the CAC strategy is an effective mean to improve system performance, especially for low to moderate traffic loads. For instance, assuming that voice toll quality is required, packet dropping probability is reduced more than $30 \%$ when primary resource occupancy information is additionally used on the CAC strategy.
\end{abstract}

Keywords- Cognitive radio networks; call admission control; VoIP; AMC; packet buffering; joint call and packet level teletraffic analysis; time-scale decomposition; on/off traffic.

\section{INTRODUCTION}

Cognitive radio (CR) technology has emerged to increase the efficiency of a largely underutilized radio spectrum. Due to the unpredictable nature of channel availability in a secondary network, researchers in early days considered only best effort services over cognitive radio networks (CRNs) without any quality-of-service (QoS) guarantees. Nonetheless, with the ever increasing popularity of cost-efficient voice over Internet protocol (VoIP) based applications, CRNs will need to support real time traffic with voice service satisfaction ${ }^{1}$. In this sense, four are the main performance indicators that characterize QoS for voice services over the Internet ${ }^{2}$ [1], [4]-[6]: 1) average endto-end delay, 2) delay variation (jitter), and 3) packet dropping probability at the packet-scale, and 4) new session blocking

\footnotetext{
${ }^{1}$ End-to-end QoS provisioning can be divided into two parts [1]: QoS in the wireless access (local) domain and QoS in the backbone domain. Since system impairments across the VoIP connection are cumulative, the required QoS at the local domain is determined by the QoS achieved at the backbone domain. After extraction of possible QoS values achieved at the backbone domain, the remaining QoS budget for the local domain is, in general, quite limited [1]-[3].

${ }^{2}$ Voice quality is acceptable if packet delay values are kept below $400 \mathrm{~ms}$, delay jitter is less than $75 \mathrm{~ms}$, packet dropping probability is less than $3 \%$, and blocking probability is less than $5 \%$ [5].
}

probability at the call-scale. When new voice sessions are admitted into an already congested network, all calls in progress will experience increased packet loss and delay. Voice quality will degrade and eventually conversation will become impossible. For these reasons, call admission control (CAC) functionality is a critical requirement for guarantee the desired QoS for CRNs under VOIP traffic.

Previous works dealing with CRNs with VoIP traffic have developed their mathematical analysis based on the assumption of either a fixed number of Secondary Users (SUs) [7]-[13] or an infinite backlog model for SUs [14]. Then, these previous studies have not considered CAC at all. On the other hand, most of the works that address CAC in CRNs make use of primary resource occupancy information (PROI) during $\mathrm{SU}$ call assignment [22]-[23]. Although, it is intuitive that such knowledge improves system performance, a comprehensive study on the impact of PROI for the admission criterion of new VoIP secondary sessions on the performance of CRNs has not been previously performed. This is the aim of our present study. Specifically, in this paper, the relevance of considering the PROI on the admission criterion of new secondary VoIP sessions in CRNs is investigated. Thus, system performance (in terms of the most relevant quality of service metrics for VoIP traffic at both call and packet level) is evaluated when PROI is used and it is compared to the case where only secondary users' occupancy information is used. From the analytical point of view, our contribution is to develop joint call and packet level teletraffic models that effectively capture relevant aspects of CRNs, VoIP traffic, and CAC strategies. Relevant system insights are found. For instance, the system's conditions under which the use of the primary network resource occupancy information yields greater improvement are identified.

\section{SYSTEM MODEL}

In a CR system, VoIP users are cognitive (or secondary) users, they occupies wireless channels transparently. Primary users (PUs) have absolute priority over SUs. It means that when VoIP secondary packet is in processing and a primary packet arrives, the PU seizes the channel. Thus, the cognitive user using this channel is interrupted and queue its packets into the buffer (the buffer length is denoted by $Q$ ). Once there are no more primary packets in the CR system, that unoccupied channel continues to dispose packets of SUs. System performance at the downlink of a wireless VoIP CR system is 
addressed, i.e., only the local domain at the receiving end is considered [1]-[3]. We assume a single base station (i.e. evolved node B or eNB in LTE vocabulary) that emulates the data transmission over $M$ shared channels. It is assumed that users requesting a VoIP session are able to connect (and stay connected up to the end of their sessions) to this base station, that is, no handoff mechanism is considered (typical in low mobility scenarios).

\section{A. Teletraffic Model}

Our proposed teletraffic model capture relevant aspects of VoIP under CRNs such as ON/OFF activity processes for individual VoIP sessions, (periodic) packet generation of individual VoIP sessions in the ON state, cognitive transmission interruptions due to primary packet arrivals, buffering of SUs' packets, adaptive modulation and coding (AMC), packet scheduling, and a CAC strategy specifically proposed for VoIP traffic over CRNs. In particular, to capture the effect of AMC, the cell is divided into $z$ coverage zones. A SU located in the $i$-th coverage is allowed to use the $i$-th data rate, denoted by $R_{b}{ }^{(i)}$. In this pure VoIP scenario, all VoIP packets have the same priority and use the same access buffering queue under the first in first out (FIFO) discipline. Also, all VoIP sessions arriving to the CRN are intended to ideally achieve the same QoS; however, a minimal level of QoS is guaranteed for all of the VoIP users. For this reason, proportional fair sharing scheduling for SUs with different link qualities is considered in our teletraffic model.

The arrival of PUs and SUs is assumed to be independent Poisson processes with arrival rate $\lambda_{P}$ and $\lambda_{S}$, respectively. It is important to notice that in this work, the average time between arrivals is considered to be in the order of seconds while the duration of the frame (inter-arrival packet time) is in the order of few milliseconds. As such, it is possible to use the time-scale decomposition technique to analyze the CRN under VoIP traffic. In Section IV, call level analysis is performed in a continuous time basis while the packet level analysis is performed in a discrete time basis. The effect of packet level on the session level is captured by connecting the resulting packet and call level queuing systems. On the other hand, call duration is also considered to be exponentially distributed with mean $1 / \mu_{\mathrm{s}}$ for cognitive users while for primary users call duration is considered generally distributed with finite mean $1 / \mu_{\mathrm{p}}$.

\section{B. Voice Traffic Model}

The ON-OFF voice traffic model proposed in [15] is adopted. In this work, we assume that the ON (talk spurt) and OFF (silent) periods are geometrically distributed with parameters $P_{\mathrm{ON}-\mathrm{OFF}}$ and $P_{\mathrm{OFF}-\mathrm{ON}}$, respectively. The parameter $P_{\text {ON-OFF }}\left(P_{\text {OFF-ON }}\right)$ represents the probability that a call that is currently in the active (silent) period turns to a silent (active) period. During the active periods, we assume that the voice samples are generated with a constant bit rate. In the VoIP call model used in this work, each voice packet is composed of protocol headers (RTP, UDP and IP) and the payload. It is considered that all users use the same voice codec, and hence both the size of VoIP packet and packet interval are identical. In our model, we consider that during the active periods the voice packets are periodically generated every $T$ seconds. Typical values used for $T$ are 10 and $20 \mathrm{~ms}$ [5], [16]. For the packet level analysis, time is structured in frames and it is considered that the frame duration is equal to $T$ seconds.

\section{CAll Admission CONTROL StRATEGIES}

In this Section, we describe the two distinct local CAC strategies with packet buffering studied in this work.

1) Strategy "S with PU". CAC based on the total number of primary and secondary users (both active and inactive sessions) in the system. In this strategy, when there are $S$ users (both primary and secondary users) in the system and a secondary user arrives, the arriving secondary user is blocked, otherwise it is accepted.

2) Strategy "S without PU”. CAC based only on the total number of secondary users (both active and inactive sessions) in the system. In this strategy, when there are $S$ secondary users in the system and a secondary user arrives, it is blocked, otherwise it is accepted.

Both strategies are basic schemes that can be easily implemented in a practical system. Indeed, the network only has to keep the count of the users in the system regardless of their current status (active or inactive).

\section{Proposed CALL-LEVEL AND PACKET-LEVEL TELETRAFFIC ANALYSIS}

In this section, the blocking and packet dropping probability analysis is performed for a VoIP service for the SUs in a CR system with AMC described above. It is assumed that active PUs in the system occupy a channel. However, the decision to accept or reject SUs is not straightforward. The CAC strategies analyzed take into account the number of active PUs in the system. The rationale behind this is that the number of SUs that can be accepted into the system should consider the amount of resources occupied by PUs in order to guarantee an acceptable QoS level. The following analysis is performed at both session and packet level. At the session level, the total number of users varies according to Poisson arrival processes and exponentially distributed session times. Therefore, a continuous time analysis is suitable. At the packet level, the total number of users in the system (both active and inactive) is considered to be fixed. As such, the probability of having a certain amount of active users and packets to be transmitted can be calculated. Since at the packet level, events occur only at the beginning of the time slots, a discrete time analysis is preferred.

\section{A. Session Level Analysis}

Now, the session level analysis is performed in order to find the performance of the system. It is important to recall that the Markov chain for the packet level analysis only considers the number of active users in different zones of the system and the number of packets to be transmitted in a given frame. This results in a very manageable analysis in terms of the number of variables due in part to the use of the decomposition methodology [17], [18]. The Markov chain described in Fig 1 is proposed in order to perform the session level analysis. It is important to recall that at session level, a continuous time analysis is proposed. Let us denote by $k_{0}$ the number of primary users in the system. Each state variable for secondary users is denoted by $k_{i}$ (for $i=1,2, \ldots, z$ ) where $z$ is the number of zones. Hence, in state $\mathbf{k}=\left[k_{0}, k_{1}, \ldots, k_{z}\right]$, there can either be one primary 
user arrival or a second user arrival at zone $i$ with rate $g_{i}(k)$. This new user arrival is expressed in vector $\mathbf{e}_{\boldsymbol{i}}$ which is a zero vector of size $z+1$ with only a 1 in position $i(i \in\{0,1,2, \ldots, z\})$. On the other hand, in state $\mathbf{k}=\left[k_{0}, k_{1}, \ldots, k_{z}\right]$, there can either be one primary user service termination or a second user that leaves the system at zone $i$ with rate $h_{i}(k)$. In this case, vector $\mathbf{e}_{i}$ is defined as in the previous case.

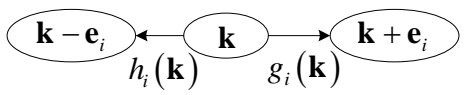

Fig. 1. Transition diagrams for the Markov chain of the CR system with AMC for IP voice traffic for the secondary users at session level.

\section{1) Strategy " $S$ " with PU occupancy information}

Note that secondary users arrive to the system according to a Poisson process. A fractional threshold on $S$ is considered, i.e., with probability $p_{\text {ses }}=S-\lfloor S\rfloor$, one secondary session is accepted when there are $\lfloor S\rfloor$ users (secondary and primary) in the system and with probability $\left(1-p_{\text {ses }}\right)$ the new secondary session is blocked. A $z+1$-dimensional birth and death process is used to obtain this probability distribution $P\left(k_{0}, k_{1}, k_{2}, \ldots, k_{z}\right)$ of secondary users in the different quality zones and the primary users.

For this strategy, the transition rates of the Markov chain described in Fig. 1 can be expressed as:

$$
\begin{gathered}
g_{i}(\mathbf{k})=\left\{\begin{array}{l}
\lambda_{P} ; k_{0} \geq 0 ; \quad k_{0}<M ; i=0 \\
p_{i} \lambda_{S} ; k_{i} \geq 0 ; \sum_{j=0}^{z} k_{j}<\lfloor S\rfloor ; i=1,2, \ldots, z \\
p_{s e s} p_{i} \lambda_{S} ; k_{i} \geq 0 ; \sum_{j=0}^{z} k_{j}=\lfloor S\rfloor ; i=1,2, \ldots, z \\
0 \quad \text { otherwise }
\end{array}\right. \\
h_{i}(\mathbf{k})= \begin{cases}k_{0} \mu_{P} ; & k_{0} \leq M ; i=0 \\
k_{i} \mu_{S} ; & \sum_{j=1}^{z} k_{j} \leq\lceil S\rceil ; i=1,2, \ldots, z \\
0 & \text { otherwise }\end{cases}
\end{gathered}
$$

where and $p_{i}$ is the probability that an arrival occurs in zone $i$ and the valid states space is given by

$$
\Omega=\left\{\mathbf{k} \mid k_{i} \geq 0, i=0,1,2, \ldots, z, k_{0} \leq M, \sum_{i=1}^{z} k_{i} \leq\lceil S\rceil\right\}
$$

From the above description, it can be seen that the system in statistical equilibrium can be described by (6)

$$
\begin{aligned}
& {\left[\sum_{i=1}^{z} g_{i}(\mathbf{k})+\sum_{i=1}^{z} h_{i}(\mathbf{k})\right] P(\mathbf{k})=} \\
& \sum_{i=1}^{z} g_{i}\left(\mathbf{k}-\mathbf{e}_{i}\right) P\left(\mathbf{k}-\mathbf{e}_{i}\right)+\sum_{i=1}^{z} h_{i}\left(\mathbf{k}-\mathbf{e}_{i}\right) P\left(\mathbf{k}-\mathbf{e}_{i}\right)
\end{aligned}
$$

Therefore, the blocking probability can be found as:

$$
P_{b}=\sum_{\mathbf{k} \mid \sum_{i=0}^{z} k_{i}>\lfloor s\rfloor} P(\mathbf{k})+\sum_{\mathbf{k} \mid \sum_{i=0}^{z} k_{i}=\lfloor s\rfloor}\left(1-p_{\text {ses }}\right) P(\mathbf{k})
$$

\section{B. Packet Level Analysis}

The mathematical analysis presented assumes that the system evolves in a discrete time basis where the unit of time is considered to be the frame, $T$. Since the value of the frame is sufficiently small compared to the inter-arrival time of users to the system, the service time in the system and the active/inactive periods, it is assumed that in a frame there can only be one user arrival to the system, or one user ending its session, or one user in OFF mode becomes active, or one in ON mode goes to stand by. The accuracy of this approximation is verified through simulation results. As such, the probability that one secondary user in zone $i(i \in\{1,2, \ldots, z\})$ goes from the ON state to the OFF state or vice versa can be calculated, respectively, as $\quad \alpha_{i}=n_{i} P_{O N \rightarrow O F F}^{i}\left(1-P_{O N \rightarrow O F F}^{i}\right)^{n_{i}-1}$ and $\beta_{i}=\left(k_{i}-n_{i}\right) P_{O F F \rightarrow O N}^{i}\left(1-P_{O F F \rightarrow O N}^{i}\right)^{k_{i}-n_{i}-1}$ where $k_{i}$ is the current total number of secondary users in zone $i$ and $n_{i}$ is the current number of active sessions of secondary users in zone $i$. Due to the Proportional Fair Sharing scheduling method, the number of packets that can be transmitted in each frame depends on the number of active users in each zone as well as the number of idle/busy primary channels. Hence, the capacity of the system (in packets per frame) at current frame when there are $k_{0}$ primary users can be described as:

$$
C=\left\lfloor\left(\frac{\sum_{i=1}^{z} R_{b}^{(i)} n_{i}}{\sum_{i=1}^{z} n_{i}}\right) T\left(M-k_{0}\right) / b\right\rfloor
$$

and the maximum capacity for the SUs is $C_{\max }=R_{b}^{1} T M / b$ where $b$ is the number of bits per packet and $T$ is the time between consecutive packets of the same user (frame duration). Building of this, the average bit rate per channel at current frame can be expressed as:

$$
\bar{R}=\frac{\sum_{i=1}^{z} R_{b}^{(i)} n_{i}}{\sum_{i=1}^{z} n_{i}}
$$

For reasons of simplicity, only two zones are considered, i.e., good and bad zones, with $n_{1}$ and $n_{2}$ users in each zone respectively at the current frame. Let $n_{3}$ be the number of packets to be transmitted during the current frame. Hence, the number of transmissions in the current frame period depends on the number of free channels detected $C$ as the number of packets in the current frame $n_{3}$ and it is given by $y=\min \left(C, n_{3}\right)$ where:

$$
C=\left\lfloor\frac{\bar{R} T\left(M-k_{0}\right)}{b}\right\rfloor
$$

where $k_{0}$ is the total number of primary users.

Packets can be lost in a frame period when the number of packets waiting transmission is higher than the available resources in the next frame period. The capacity at the next 
frame period depends on the free channel in the previous frame as the number of active secondary users in the next frame period, and is described by:

$$
\bar{C}=\left\lfloor\left(\frac{\sum_{i=1}^{z} R_{b}^{(i)} \bar{n}_{i}}{\sum_{i=1}^{z} \bar{n}_{i}}\right) T\left(M-k_{0}\right) / b\right\rfloor
$$

where $\bar{n}_{i}$ and $\bar{C}$ denote, respectively, the values of the total number of secondary users users in $i$-th zone and the capacity at the next frame period.

Hence, the total number of packets lost from the current frame to the next frame can be computed as follows:

$$
L_{p}=\max \left(0, n_{1}+n_{2}+n_{3}-y-\bar{C}-Q\right)
$$

where $Q$ is the queue length in VoIP packets. From this, it can be seen that the number of secondary users' packets to be transmitted at the next frame period is given by:

$$
n_{3}=n_{3}+x-L_{p}
$$

where $x=n_{1}+n_{2}-y$.

The aforementioned system can be modeled as a discretetime Markov chain $W=\left(n_{1}, n_{2}, n_{3}\right)$ with valid states spaces

$$
\Omega=\left\{\begin{array}{l}
\mathbf{k} \mid 0 \leq k_{0} \leq M ; 0 \leq \sum_{i=1}^{z} k_{i} \leq\lceil S\rceil \\
\mathbf{n} \mid 0 \leq n_{i} \leq k_{i}, \quad 0 \leq n_{z+1} \leq C_{\max }+Q, i=1,2, \ldots, z
\end{array}\right\}
$$

where $S$ is calculated in order to achieve a blocking probability of 0.05 for secondary users. Also, a primary user is blocked when primary users are using the $M$ channels. $W$ is a discrete time homogeneous Markov chain, with initial state $W=(0,0,0)$. Starting from state $\left(n_{1}, n_{2}, n_{3}\right)$ the possible transitions are as follows:

- To state $\left(n_{1}, n_{2}, n_{3}+x-L_{p}\right)$ with probability

$a_{0}=\left(1-P_{\text {ON } \rightarrow \text { OFF }}^{1}\right)^{n_{1}}\left(1-P_{\text {ON } \rightarrow O F F}^{2}\right)^{n_{2}}\left(1-P_{\text {OFF } \rightarrow O N}^{1}\right)^{k_{1}-n_{1}}\left(1-P_{O F F \rightarrow O N}^{2}\right)^{k_{2}-n_{2}}$

for $0 \leq n_{3}+x+L_{p} \leq C_{\max }+Q$ when there are no changes in the number of active users in the system and only the packets at the beginning of the frame are transmitted and possibly lost.

- To state $\left(n_{1}+1, n_{2}, n_{3}+x-L_{p}\right)$ with probability

$a_{1}=\left(1-P_{\mathrm{ON} \rightarrow \mathrm{OFF}}^{1}\right)^{n_{1}} \beta_{1}\left(1-P_{\mathrm{ON} \rightarrow \mathrm{OFF}}^{2}\right)^{n_{2}}\left(1-P_{\mathrm{OFF} \rightarrow \mathrm{ON}}^{2}\right)^{k_{2}-n_{2}}$

for $n_{1}<k_{1} ; 0 \leq n_{3}+x+L_{p} \leq C_{\max }+Q$ when a user in the zone 1 becomes active.

- To state $\left(n_{1}-1, n_{2}, n_{3}+x-L_{p}\right)$ with probability

$a_{2}=\alpha_{1}\left(1-P_{O F F \rightarrow O N}^{1}\right)^{k_{1}-n_{1}}\left(1-P_{O N \rightarrow O F F}^{2}\right)^{n_{2}}\left(1-P_{O F F \rightarrow O N}^{2}\right)^{k_{2}-n_{2}}$

for $n_{1}>0 ; 0 \leq n_{3}+x+L_{p} \leq C_{\max }+Q$ when a user in zone 1 goes to the OFF mode.
- To state $\left(n_{1}, n_{2}+1, n_{3}+x-L_{p}\right)$ with probability $a_{3}=\left(1-P_{\mathrm{ON} \rightarrow \mathrm{OFF}}^{1}\right)^{n_{1}}\left(1-P_{\mathrm{OFF} \rightarrow \mathrm{ON}}^{1}\right)^{k_{1}-n_{1}}\left(1-P_{\mathrm{ON} \rightarrow \mathrm{OFF}}^{2}\right)^{n_{2}} \beta_{2}$ for $n_{2}<k_{2} ; 0 \leq n_{3}+x+L_{p} \leq C_{\max }+Q$ when there is one more active user in zone 2 of the system.

- To state $\left(n_{1}, n_{2}-1, n_{3}+x-L_{p}\right)$ with probability $a_{4}=\left(1-P_{O N \rightarrow O F F}^{1}\right)^{n_{1}}\left(1-P_{O F F \rightarrow O N}^{1}\right)^{k_{1}-n_{1}} \alpha_{2}\left(1-P_{O F F \rightarrow O N}^{2}\right)^{k_{2}-n_{2}}$ for $n_{2}>0 ; 0 \leq n_{3}+x+L_{p} \leq C_{\max }+Q$ when there is one active user in zone 2 that goes to the OFF mode.

- To state $\left(n_{1}+1, n_{2}+1, n_{3}+x-L_{p}\right)$ with probability $a_{5}=\left(1-P_{O N \rightarrow O F F}^{1}\right)^{n_{1}} \beta_{1}\left(1-P_{O N \rightarrow O F F}^{2}\right)^{n_{2}} \beta_{2}$ for $n_{1}<k_{1} ; n_{2}<k_{2} ; 0 \leq n_{3}+x+L_{p} \leq C_{\max }+Q$ when there is one new user in both zones, i.e., 1 and 2 .

- To state $\left(n_{1}-1, n_{2}+1, n_{3}+x-L_{p}\right)$ with probability $a_{6}=\alpha_{1}\left(1-P_{O F F \rightarrow O N}^{1}\right)^{k_{1}-n_{1}}\left(1-P_{O N \rightarrow O F F}^{2}\right)^{n_{2}} \beta_{2}$

for $n_{1}>0 ; n_{2}<k_{2} ; 0 \leq n_{3}+x+L_{p} \leq C_{\max }+Q$ when one user goes to the OFF mode in zone 1 and one user becomes active in zone 2 .

- To state $\left(n_{1}+1, n_{2}-1, n_{3}+x-L_{p}\right)$ with probability $a_{7}=\left(1-P_{\mathrm{ON} \rightarrow \mathrm{OFF}}^{1}\right)^{n_{1}} \beta_{1} \alpha_{2}\left(1-P_{\mathrm{OFF} \rightarrow \mathrm{ON}}^{2}\right)^{k_{2}-n_{2}}$

for $n_{1}<k_{1} ; n_{2}>0 ; 0 \leq n_{3}+x+L_{p} \leq C_{\max }+Q$ when a user in zone 1 goes to the ON mode while a user in zone 2 goes to the OFF mode.

- To state $\left(n_{1}-1, n_{2}-1, n_{3}+x-L_{p}\right)$ with probability $a_{8}=\alpha_{1}\left(1-P_{O F F \rightarrow O N}^{1}\right)^{k_{1}-n_{1}} \alpha_{2}\left(1-P_{O F F \rightarrow O N}^{2}\right)^{k_{2}-n_{2}}$

for $n_{1}>0 ; n_{2}>0 ; 0 \leq n_{3}+x+L_{p} \leq C_{\max }+Q$ when there is one user that goes to the OFF mode in both zones 1 and 2 .

Note that the effect of the primary users is given trough the variable $n_{3}$ which is calculated using the capacity of the system described by (1). This chain is numerically solved using the Gauss-Seidel method by considering the set of lineal equations given by $\Pi \mathbf{P}=\Pi$ where $\Pi$ is the steady state probabilities given by $\left[\Pi_{\{0,0,0 \mid \mathbf{k}\}}, \Pi_{\{0,0,1 \mid \mathbf{k}\}}, \ldots, \Pi_{\left\{n_{1}, n_{2}, n_{3} \mid \mathbf{k}\right\}}, \ldots\right]$ and $\mathbf{P}$ is the transition probabilities matrix composed by the valid transitions in the system described above.

Using (4), it is possible to find the average number of packets lost per time slot as:

$$
E\left\{L_{p}(\mathbf{n} \mid \mathbf{k})\right\}=\sum_{n_{1}=0}^{k_{1}} \sum_{n_{2}=0}^{k_{2}} \ldots \sum_{n_{z}=0}^{k_{z}} \sum_{n_{z+1}=0}^{C_{\max }+Q} L_{p}(\mathbf{n} \mid \mathbf{k}) \Pi(\mathbf{n} \mid \mathbf{k})
$$


And the average number of packets generated per frame is calculated as:

$$
E\left\{\sum_{i=1}^{z} n_{i} \mid \mathbf{k}\right\}=\sum_{n_{1}=0}^{k_{1}} \sum_{n_{2}=0}^{k_{2}} \ldots \sum_{n_{z}=0}^{k_{z}} \sum_{n_{z+1}=0}^{C_{\max }+Q}\left(\sum_{i=1}^{z} n_{i}\right) \Pi(\mathbf{n} \mid \mathbf{k})
$$

Hence, packet dropping probability, $P_{d}$, is is found by considering all the possible values of $k_{0}, k_{1}, \ldots, k_{z}$. Then:

$$
P_{d}=\frac{\sum_{k_{0}=0}^{M} \sum_{k_{1}=0}^{\lceil S\rceil} \sum_{k_{2}=0}^{\lceil s\rceil-k_{1}} \cdots \sum_{k_{z}=0}^{\lceil s\rceil-\sum_{i=1}^{z-1} k_{i}} E\left\{L_{p}(\mathbf{n} \mid \mathbf{k})\right\} P(\mathbf{k})}{\sum_{k_{0}=0}^{M} \sum_{k_{1}=0}^{\lceil S\rceil} \sum_{k_{2}=0}^{\lceil s\rceil-k_{1}} \cdots \sum_{k_{z}=0}^{\lceil s\rceil-\sum_{i=1}^{z-1} k_{i}} E\left\{\sum_{i=1}^{z} n_{i} \mid \mathbf{k}\right\} P(\mathbf{k})}
$$

1) Strategy " $S$ " without $P U$ occupancy information

When the activity of the PU's is not considered, the traffic analysis shown above is affected in the system capacity expression. In this case the total number of resources available is always $M$ no matter the number of PU's active sessions in the system. So the equation (3) is rewritten as

$$
C=\left\lfloor\frac{\bar{R} T M}{b}\right\rfloor
$$

\section{NUMERICAL RESUltS}

The goal of the numerical evaluations presented in this section is to quantify system performance improvement due to the use of primary resource occupancy information (PROI) on the admission threshold of a CRN under VoIP traffic. System performance is evaluated in terms of packet dropping probability and mean packet delay, for a target session blocking probability (i.e., 5\%). Unless otherwise specified, the following values of the system parameters were used in the plots of this section. Voice activity factor 0.5 (i.e., $P_{\text {ON-OFF }}=P_{\text {OFF-ON }}=$ $1 / 1800) ; R_{b_{1}}=4 \mathrm{kbps} ; R_{b_{2}}=2 \mathrm{kbps} ; T_{f}=1 \mathrm{~s}$; packet size $b=2 \mathrm{~kb}$; total number of channels $M=4 ; N_{s}=2$ slots per frame; $P_{s}=1 / 18000 ; \mu_{p}=1 / 18000$; traffic load of PUs (in order to obtain a primary blocking probability equal $0.5 \%$ ) $a_{p}=0.702$ Erlang.

Fig. 2 shows analytical and simulation results for the packet dropping probability as function of secondary traffic load with queue size as parameter for strategy $\mathrm{S}$ with and without PROI. As expected, packet dropping probability is a monotonically increasing function of the secondary traffic load. Table I shows the percentage reduction on the packet dropping probability of strategy S with PU' occupation information relative to Strategy S without PU' occupation information. In general, from Fig. 2 and Table I, good agreement between analytical and simulation results is observed. Even though the gap between the simulation and the analytic results increase with $a_{s}$ in Fig. 2, the relative difference between simulation and analytical results, in general, decreases or remains at similar values as $a_{s}$ increases. The most relevant result that can be extracted from Fig. 2 and Table I is that the performance of strategy S with PU' occupation information outperforms strategy S without PU' occupation information. This is especially true for values of packet dropping probability that corresponds to toll quality. For instance, assuming that voice toll (business) quality is required and $Q=200$, packet dropping probability is reduced more than $30 \%(12 \%)$ when primary resource occupancy information is used on the strategy S. Similar values are obtained when queue size of 50 is considered. This behavior can be explained as follows. Due to the fact that strategy S with PU' occupancy information uses the effective number of available radio resources at the session request, a more effective QoS protection of ongoing sessions can be performed relative to the strategy $\mathrm{S}$ without PU' occupancy information.

Fig. 3 shows mean packet delay as function of secondary traffic load with queue size as parameter for strategies $\mathrm{S}$ with and without PROI. As expected, packet delay is a monotonically increasing function of secondary traffic load. A relevant result that can be extracted from Fig. 3 is that the performance of strategies S with PU' occupation information outperforms strategy S without PU' occupation information. This is especially true for low and moderate values of secondary traffic load. For instance, assuming that secondary traffic load equals 1 (3) Erlang(s) and $Q=200$, mean packet delay is reduced $27 \%(8 \%)$ when primary resource occupancy information is used on the strategy $\mathrm{S}$.

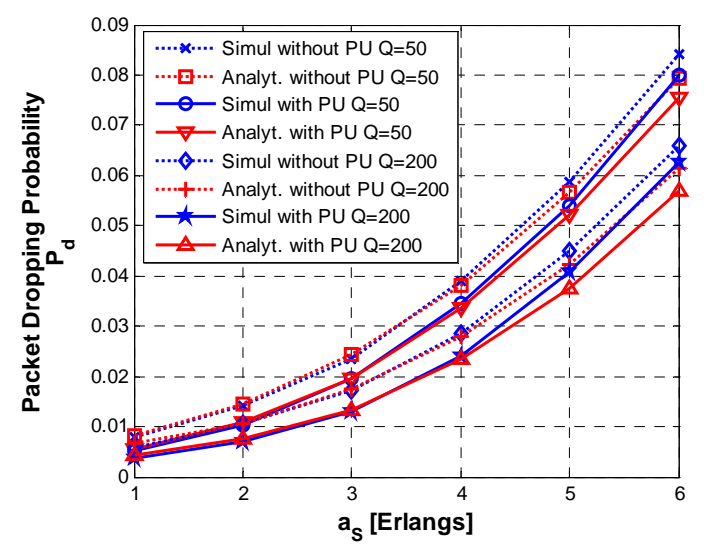

Fig. 2. Packet dropping probability as function of secondary traffic load with queue size as parameter for strategies $\mathrm{S}$ with and without primary channel occupancy information.

TABLE I PACKET DROPPING PROBABILITY REDUCTION DUE TO THE USE OF

\begin{tabular}{|c|c|c|c|c|c|}
\hline \multirow[b]{2}{*}{$a_{S}$} & \multicolumn{5}{|c|}{ Packet dropping probability } \\
\hline & $\begin{array}{l}\text { Analytical } \\
\text { with PU }\end{array}$ & $\begin{array}{l}\text { Simulation } \\
\text { with } P U\end{array}$ & $\begin{array}{l}\text { Analytical } \\
\text { without } \\
\text { PU }\end{array}$ & $\begin{array}{l}\text { Simulation } \\
\text { without } \\
\text { PU }\end{array}$ & $\begin{array}{l}\text { Reduction (comparing } \\
\text { without and with PU } \\
\text { results) }\end{array}$ \\
\hline \multicolumn{6}{|c|}{$Q=50$} \\
\hline 1 & 0.0055 & 0.0051 & 0.0083 & 0.0078 & $34.4 \%$ \\
\hline 2 & 0.0108 & 0.0102 & 0.0146 & 0.0141 & $27.2 \%$ \\
\hline 3 & 0.0197 & 0.0197 & 0.0243 & 0.0235 & $16.1 \%$ \\
\hline 4 & 0.0337 & 0.0344 & 0.0382 & 0.0391 & $12.1 \%$ \\
\hline 5 & 0.0520 & 0.0539 & 0.0566 & 0.0586 & $8.1 \%$ \\
\hline 6 & 0.0754 & 0.0799 & 0.0794 & 0.0842 & $5.1 \%$ \\
\hline \multicolumn{6}{|c|}{$Q=200$} \\
\hline 1 & 0.0045 & 0.0038 & 0.0067 & 0.0059 & $35 \%$ \\
\hline 2 & 0.0075 & 0.007 & 0.0107 & 0.0106 & $34.1 \%$ \\
\hline 3 & 0.013287 & 0.0131 & 0.0175 & 0.0172 & $24.2 \%$ \\
\hline 4 & 0.0233 & 0.0241 & 0.0279 & 0.0286 & $15.8 \%$ \\
\hline 5 & 0.0376 & 0.0409 & 0.0424 & 0.0449 & $8.9 \%$ \\
\hline 6 & 0.057 & 0.0626 & 0.0614 & 0.066 & $5.1 \%$ \\
\hline
\end{tabular}
PRIMARY OCCUPANCY RESOURCE INFORMATION IN THE ADMISSION THRESHOLD OF THE S STRATEGY. 


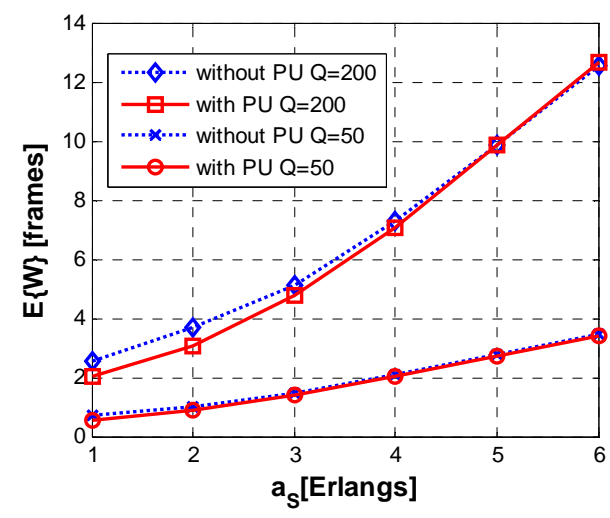

Fig. 3. Mean packet delay as function of secondary traffic load with queue size as parameter for strategies $\mathrm{S}$ with and without primary channel occupancy information.

In summary, numerical results clearly show that including PROI on the CAC strategy is an effective mean to improve system performance in CRNs under VoIP traffic. However, when the packet dropping probability is required to be low, the gain in performance obtained by the use of the PROI is considerable higher than when the packet dropping probability is high. As such, the impact of the use of the PROI is not always important.

\section{CONCLUSIONS}

In this paper, the relevance of considering the primary network resource occupancy information for the admission criterion of new secondary sessions on the performance of CRNs with VoIP traffic was investigated. To this end, joint call and packet level teletraffic models that effectively capture relevant aspects of CRNs under VoIP traffic were developed. The developed teletraffic models are based on time-scale decomposition technique and employs conventional both continuous (for session level analysis) and discrete (for packet level analysis) time Markov chain tools. Numerical results show that including primary resource occupancy information on the CAC strategy is especially attractive to improve performance of the cognitive radio network for low to moderate offered traffic loads. Packet dropping reduction greater than $30 \%$ is observed.

\section{REFERENCES}

[1] K. Kassev, Y. Mihov, A. Kalaydzhieva, and B. Tsankov, "A new paradigm of CAC dimensioning for VoIP Traffic over wireless access networks," in Proc. 4th Intl. Conf. on Digital Society 2010, St. Maarten, Netherlands Antilles, Feb. 2010, pp. 54-59.

[2] E.B. Rodriguez, F.R.P. Cavalcanti, S. Wanstedt, "QoS-Driven adaptive congestion control for voice over IP in multiple service wireless cellular networks," IEEE Commun. Mag., vol. 46, no. 1, pp. 100-107, Jan. 2008.

[3] S. Maniatis, E. G. Nikolouzou, and I. Venieris, "End-to-end QoS specification issues in the converged all-IP wired and wireless environment," IEEE Commun. Mag., vol 42, no. 6, pp. 80-86, Jun. 2004.

[4] S.L. Castellanos-López, F.A. Cruz-Pérez, M.E. Rivero-Ángeles, and G. Hernández-Valdez, "Joint call and packet level performance analysis of CAC strategies for VoIP traffic in wireless networks," in Proc. IEEE Globecom'2011, Houston, Texas, Dec. 2011.

[5] S. Karapantazis, F.-N- Pavlidou, "VoIP: A comprehensive survey on a promising technology," Comp. Networks, vol. 53, pp. 2050-2090, 2009.
[6] T. Uhl, "Quality of service in VoIP communication, Int. J. Electron. Commun, vol. 58, no. 3, pp 178-182, 2004.

[7] L. Jiang, T. Jiang, Z. Wang, and X. He, "Capacity analysis of VoIP service in cognitive radio system," in Proc. WiCOM'2010, Chengdu, China, Sep. 2010.

[8] Z. Wang, T. Jiang, L. Jiang, X. He, "VoIP capacity analysis in cognitive radio system with single/multiple channels," in Proc. WiCOM'2010, Chengdu, China, Sept. 2010.

[9] H. Lee and D.-H. Cho, "VoIP capacity analysis in cognitive radio systems," IEEE Commun. Letters, vol. 13, no. 6, pp. 393-395, June 2009.

[10] H. Lee and D.-H. Cho, "Capacity improvement and analysis of VoIP service in a cognitive radio system," IEEE Trans. Veh. Technol., vol. 59, pp. 1646-1651, May 2010.

[11] S. Gunawardeba and W. Zhuang, "Voice capacity of cognitive radio networks," IEEE ICC 2010, Cape Town, South Africa, May 2010.

[12] S. Gunawardeba and W. Zhuang, "Capacity analysis and call admission control in distributed cognitive ratio networks," IEEE Trans Wireless Commun., vol. 10, no.9, pp. 3110-3120, Sep. 2011.

[13] P. Wang, D. Niyato, and H. Jiang "Voice-service capacity analysis for cognitive radio networks," IEEE Trans. Veh. Technol., vol. 59, no. 4, pp. 1779-1790, May 2010.

[14] F. Wang, J. Zhu, J. Huang, and Y. Zhao, "Admission control and channel allocation for supporting real-time applications in cognitive radio networks," in Proc. IEEE Globecom'2010, Miami, FL, Dec. 2010.

[15] P. T. Brady, "A model for generating ON-OFF speech patterns in twoway conversations," Bell Syst. Technol. Journal, vol. 48, no. 7, pp. 2445-2472, Sep. 1969.

[16] Q. Bi, S. Vitebsky, Y. Yang, Y. Yuan, and Q. Zhang, "Performance and capacity of cellular OFDMA systems with voice-over-IP traffic," IEEE Trans. Veh. Technol., vol. 57, no. 6, pp. 3641-3652, Nov. 2008.

[17] D. Mitra, M. I. Reiman, and J. Wang, "Robust dynamic admission control for unified cell and call QoS in statistical multiplexers," IEEE J. Select. Areas Commun., vol. 16, pp. 692-707, June 1998.

[18] S. Ghani, M. Schwartz, "A decomposition approximation for the analysis of voice/data integration," IEEE Trans. Commun., vol. 42, no. 7, Jul. 1994, pp. 2441-2452.

[19] S.L. Castellanos-López, F.A. Cruz-Pérez, M. Rivero-Angeles, and G. Hernández-Valdez, "Simplified joint call and packet level teletraffic analysis of CAC strategies for VoIP traffic in wireless networks," accepted for its publication in IEEE WCNC'2012, Paris, France, April 2012.

[20] ITU-R Recommendation G.114, General characteristics of international telephone connections and international telephone circuits: one-way transmission time, February 1996.

[21] ETSI TIPHON, End-to-end quality of service in TIPHON systems; Part II: Definition of Quality of Service (QoS) classes, TS 101 329-2, July 2000

[22] D. Pacheco-Paramo V. Pla, and J. Martinez-Bauset, "Optimal admission control in cognitive radio networks," in Proc. IEEE CROWNCOM'2009, Hannover, Germany, Jun. 2009.

[23] Ming Huang, Rong Yu, Yan Zhang, "Call Admission Control with SoftQoS Based Spectrum Handoff in Cognitive Radio Networks," in Proc. ACM IWCMC'2009, Leipzig, Germany, June 2009, pp. 1067-1072. 